\title{
Qiliqiangxin Protects Against Cardiac Ischemia-Reperfusion Injury via Activation of the mTOR Pathway
}

\author{
Yonglan Zhou ${ }^{a}$ Hongyi Fang ${ }^{a}$ Shenghui Lin ${ }^{a, b}$ Shutong Shen ${ }^{a}$ Lichan Tao ${ }^{a}$ \\ Junjie Xiao a,c,d Xinli Lia \\ aDepartment of Cardiology, The First Affiliated Hospital of Nanjing Medical University, Nanjing,

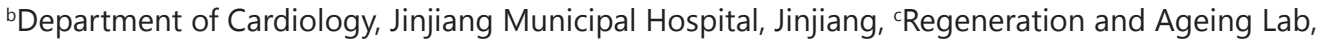 \\ Experimental Center of Life Sciences, School of Life Science, Shanghai University, Shanghai, dShanghai \\ Key Laboratory of Bio-Energy Crops, School of Life Science, Shanghai University, Shanghai, China
}

\author{
Key Words \\ Qiliqiangxin • Cardiac ischemia-reperfusion injury • mTOR
}

\begin{abstract}
Background/Aims: Qiliqiangxin (QL) has been used for the treatment of chronic heart failure in China. Accumulating evidence suggests QL's cardio-protective effects on continuous myocardial ischemia. However, it is unclear whether QL has beneficial effects on cardiac ischemia-reperfusion (I/R) injury. Methods: A mouse model of cardiac I/R was established by ligation of the left anterior descending coronary artery for 45 minutes followed by reperfusion. The mice were treated with QL for three days before surgery and continually after I/R. Triphenyltetrazolium chloride staining, echocardiography and Masson's trichrome staining were used to determine infarct size, cardiac function, and fibrosis, respectively. Expression levels of phospho-mTOR (Ser2448), mTOR, phospho-4EBP (Ser65), 4EBP, phospho-Akt (Ser473) and Akt were detected by Western blotting. Results: At 1 day after $I / R, Q L$ treatment significantly reduced the infarct size of mice exposed to I/R. At 7 days after $I / R$, mortality was reduced in QL treated animals in comparison with the control group. In addition, QL treated mice showed increased left ventricular ejection fraction (LVEF) and left ventricular fractional shortening (LVFS) at 1 and 7 days after I/R. In agreement, Masson's trichrome staining demonstrated that interstitial fibrosis was less pronounced in QL treated mice compared with controls, suggesting that adverse left ventricular remodeling is attenuated in QL treated mice. Moreover, western blotting analysis demonstrated that QL activated the mTOR pathway, while mTOR inhibition via Rapamycin abolished the protective effects of QL against I/R injury. Conclusion: This study suggests that QL attenuates the progression of cardiac remodeling after I/R likely via mTOR activation. This represents a new application for QL in the prevention of I/R injury.
\end{abstract}

Y. Zhoua, H. Fang and S. Lin contributed equally to this work.

Dr. Xinli Li and Dr. Junjie Xiao

KARGER 125
Department of Cardiology, The First Affiliated Hospital of Nanjing Medical University, 300 Guangzhou Road, Nanjing 210029, (China) and Regeneration and Ageing Lab, Experimental Center of Life Sciences, School of Life Science, Shanghai University, 333 Nan Chen Road, Shanghai 200444, (China)

E-Mail xinli3267_nj@hotmail.com and E-Mail junjiexiao@live.cn 


\begin{tabular}{|c|c|c|}
\hline Cellular Physiology & Cell Physiol Biochem 2015;37:454-464 & \\
\hline and Biochemistry & $\begin{array}{l}\text { DOI: 10.1159/000430368 } \\
\text { Published online: August 28, } 2015\end{array}$ & $\begin{array}{l}\text { O } 2015 \text { S. Karger AG, Basel } \\
\text { www.karger.com/cpb }\end{array}$ \\
\hline
\end{tabular}

\section{Introduction}

Acute myocardial infarction (MI) caused by coronary artery occlusion is a common cause of cardiac dysfunction and heart failure (HF) [1]. Early restoration of myocardial perfusion is the primary goal of initial treatment for patients with acute MI [1]. Currently, advances in pharmacological therapy and percutaneous coronary intervention can restore coronary flow in a majority of patients within a short time after the onset of symptoms [2]. However, restoring blood flow itself may extend cardiac injury, a phenomenon referred to as reperfusion injury $[3,4]$. Adverse left ventricular (LV) remodeling after acute MI, characterized by LV dilatation and fibrosis, is a critical determinant of the subsequent development of HF [5]. LV remodeling is also an important pathophysiological feature in ischemia-reperfusion (I/R) injury [6]. Therefore, identifying pathways that effectively protect against I/R injury and/or adverse remodeling could lead to novel therapeutic approaches to mitigating $\mathrm{LV}$ remodeling and $\mathrm{HF}$ after acute MI.

The mammalian target of rapamycin (mTOR) is an important mediator of the insulinPI3K-Akt axis in multiple organs, including the heart [7]. mTOR forms two functional complexes: rapamycin-sensitive mTOR complex 1 (mTORC1) and rapamycin-insensitive mTOR complex 2 (mTORC2) [8]. mTORC1 activates p70S6 kinase that phosphorylates the ribosomal protein $\mathrm{S} 6$, and inhibits the binding of 4E-binding protein 1 (4EBP) to eukaryotic translation initiation factor $4 \mathrm{E}$, resulting in the promotion of translation [9]. mTORC2 activates Akt, a key regulator of cardiomyocyte survival, by phosphorylation at Ser473 $[10,11]$. Previous studies using pharmacological mTOR inhibitors, including rapamycin, in both in vivo and ex vivo models of MI have found discrepant effects. Some reports have demonstrated beneficial effects of mTOR activation in I/R models [12-16]; conversely, another report described cardio-protective effects of mTOR inhibition [10]. Thus, the role of mTOR in cardiac function and LV remodeling after I/R injury remains undefined.

Qiliqiangxin capsule (QL) is a Chinese patent drug that was shown to be effective and safe in our recent clinical trial for the treatment of patients with chronic heart failure [17]. QL includes 11 Chinese herbs, with Radix Astragali and Aconite Root containing the main active constituents [18]. Radix Astragali is known to attenuate adverse cardiac remodeling after MI and hypertrophy [19]. However, whether QL has a role in cardiac function and LV remodeling after I/R injury remains unknown. Therefore, the present study aimed to assess QL's beneficial effects on I/R injury in mice and explore potential mechanisms.

\section{Materials and Methods}

Animals

Eight to ten week old C57BL/6 male mice (20-25g) were obtained from Laboratory Animal Center of Nanjing Medical University and housed with 12-hour dark/light cycle and free access to food in accordance with the regulations on mouse welfare. This study complied with standards for the Care and Use of Laboratory Animals (Laboratory Animal Center of Nanjing Medical University), and all procedures were approved by the Ethics Review of Lab Animal Use Application of Nanjing Medical University.

\section{In vivo $I / R$ model}

The I/R model was established according to previously reported studies [20-23]. Briefly, animals were anesthetized i.p. with ketamine and sevoflulene, intubated, and ventilated. A left thoracotomy was performed, and the left anterior descending coronary artery (LAD) was ligated using 7-0 silk sutures with a section of PE-10 tubing placed over the LAD, $1 \mathrm{~mm}$ from the tip of the normally positioned left atrium. After 45 -min ischemia, the LAD ligature was released and reperfusion was visually confirmed. During the procedure, body temperature was maintained with a $37^{\circ} \mathrm{C}$ warming plate. The operated mice were euthanized at 1 and 7 days after I/R for infarction size, fibrotic area, and signaling pathway assessment. Sham operations were carried in a similar fashion, but without suturing the LAD. 


\section{Cellular Physiology Cell Physiol Biochem 2015;37:454-464 \begin{tabular}{l|l|l}
\hline DOI: 10.1159/000430368 & C 2015 S. Karger AG, Basel
\end{tabular} \begin{tabular}{l|l|l|l|l} 
and Biochemistry Published online: August 28, 2015 & www.karger.com/cpb
\end{tabular} \\ Zhou et al.: Qiliqiangxin Protects Cardiac Ischemia-Reperfusion Injury}

\section{In vivo $Q L$ and Rapamycin Treatment}

QL was provided by Shijiazhuang Yiling Pharmaceutic (Hebei, China). The herbal drugs were authenticated and standardized with marker compounds according to the Chinese Pharmacopoeia 2005 (National Pharmacopoeia Committee, 2005). The QL powder was dissolved in normal saline (NS). Mice were randomized for intragastric treatment with QL $(0.5 \mathrm{~g} / \mathrm{kg} /$ day, $\mathrm{n}=25)$ or NS $(\mathrm{n}=25)[17,24,25]$. Sham operated mice were also given QL $(0.5 \mathrm{~g} / \mathrm{kg} /$ day, $\mathrm{n}=10)$ or $\mathrm{NS}(\mathrm{n}=10)$. All mice were treated for three days before surgery and afterward, until euthanasia. In order to investigate if mTOR activation is required for the protective effects of QL in I/R injury, Rapamycin (a specific mTOR inhibitor, $5 \mathrm{mg} / \mathrm{kg}$ ) was injected via the tail vein 10 min before cardiac I/R in the I/R+QL group.

\section{Measurement of Myocardial Infarction Size}

At 24 hours after I/R, mice were anesthetized i.p. with 0.4 to $0.75 \mathrm{mg} / \mathrm{g}$ tribromoethanol. Then, 1 $\mathrm{ml}$ Evans blue $(0.01 \mathrm{~g} / \mathrm{ml}$; BioSharp, China) was slowly injected into inferior vena and the heart removed immediately $[26,27]$. After storage for $10 \mathrm{~min}$ at $-20^{\circ} \mathrm{C}$, the heart was cut into 5 or 6 transverse slices $(1 \mathrm{~mm}$ thickness) across the long axis. Then, the slices were stained with $1 \%$ triphenyltetrazolium chloride (TTC, Amresco, USA) in citrate buffer solution ( $\mathrm{ph}=7.4$ ) for $10 \mathrm{~min}$ at $37^{\circ} \mathrm{C}$, to delineate the area at risk (AAR) [2023]. Afterwards, all slices were fixed with $4 \%$ paraformaldehyde, photographed and analyzed. As previously reported $[28,29]$, the infarct area (IA) appeared white, while the non-infarct but at risk area was red. The final infarct size was expressed as the ratio of IA to AAR, calculated by computerized planimetry (Image J, version 1.44, NIH, Bethesda, MD).

\section{Echocardiography Measurement}

Cardiac function was evaluated on a high-frequency ultrasound system Vevo2100 (VisualSonics Inc, Toronto, ON, Canada) with a $30 \mathrm{MHz}$ central frequency scan head at 1 and 7 days after I/R operation. Mice were anesthetized with 1-2\% isoflurane vapor in a 1:1 oxygen mixture via a nose cone on a heating pad to maintain normothermia. The left ventricular ejection fraction (LVEF; \%) and left ventricular fractional shortening (LVFS; \%) were assessed according to the guidelines accompanying the Vevo2100 system.

\section{Histological Analysis}

To evaluate the morphological changes and the extent of cardiac fibrosis, the hearts were harvested at 7 days after I/R, washed in PBS, fixed in 4\% paraformaldehyde overnight and embedded in paraffin. Each heart was cut into sections of $4 \mu \mathrm{m}$-thick and stained with hematoxylin and eosin (H\&E) and Masson's trichrome. Micrographs were acquired on a Nikon light microscope (Nikon, Japan).

\section{Western Blotting Analysis}

At 24 hours after reperfusion, total protein was obtained from left ventricular myocardial tissues after extraction with the RIPA lysis buffer (P0013B, Beyotime, China). Protein concentration was measured by the Pierce $^{\mathrm{TM}}$ BCA Protein Assay Kit (Thermo Scientific, USA). A total of $60 \mu \mathrm{g}$ total protein were electrophoresed and separated on $4-12 \%$ SDS-PAGE, and transferred onto nitrocellulose membranes (Millipore, US). The membranes were blocked with $5 \%$ skim milk at room temperature for an hour, and incubated overnight at $4^{\circ} \mathrm{C}$ with primary antibodies raised in rabbits against mTOR (1:1000), phospho-mTOR (Ser2448, 1:1000), 4EBP (1:1000), phospho-4EBP (Ser65, 1:1000), Akt (1:1000), phospho-Akt (Ser473, 1:1000) purchased from Cell Signaling Technology (USA). Then, the membranes were incubated with HRP-conjugated secondary antibodies (1:2000; Cell Signaling Technology, USA) at room temperature for two hours. The signals were detected with a SuperSignal ECL kit (Thermo, USA) in a Western blotting detection system (Bio-Rad, CA, USA). HRP-conjugated monoclonal mouse anti-GAPDH (1:5000; KANGCHEN, China) was used to quantify GAPDH levels. Results were expressed as density values normalized to GAPDH.

\section{Statistical Analyses}

Data were presented as mean \pm standard error of mean (SEM). Group differences were analyzed by a two-tailed Student's $t$-test. For multiple comparisons, one-way ANOVA with Bonferroni post hoc test was used. The overall mouse survival after I/R was evaluated using Kaplan-Meier curves and compared by log-rank test. All statistical analyses were conducted using SPSS 15.0 or GraphPad Prism 5. P $<0.05$ was considered statistically significant. 
Fig. 1. Percent survival at 1 week after I/R. No mouse died in the Sham+NS and Sham + QL groups. Kaplan-Meier analysis showed significantly lower mortality in QL treated mice after I/R compared with the untreated animals.

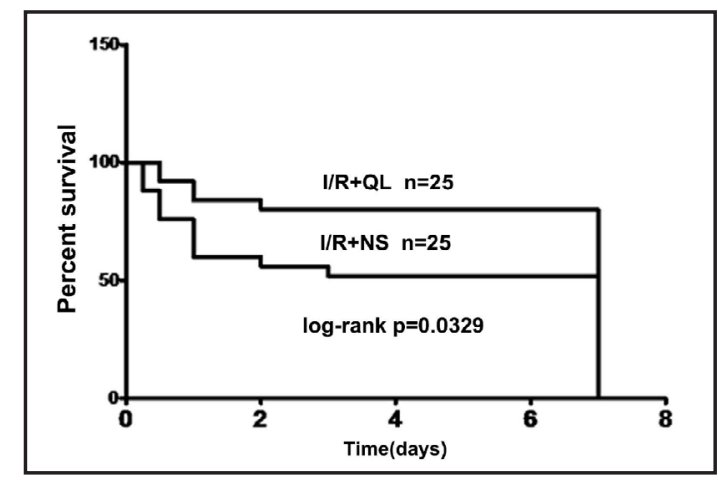

Fig. 2. QL preserves cardiac function after I/R. (A) Representative M-mode images of operated mice at pre-I/R, 24 hours and 7 days after I/R. (B-C) LVEF and LVFS analysis at pre-I/R, 24 hours and 7 days after I/R. ${ }^{* * *} \mathrm{P}<0.001$ versus sham- operated mice, $++\mathrm{P}<0.01 \mathrm{I} / \mathrm{R}+\mathrm{QL}$ versus $\mathrm{I} / \mathrm{R}+\mathrm{NS},+\mathrm{P}<0.05 \mathrm{I} / \mathrm{R}+\mathrm{QL}$ versus I/R+NS. (D) Heart weight/body weight (HW/ BW) ratios at 7 days after I/R $(n=10$, sham-operated mice; $n=20$, QL treated mice; $n=13$, untreated mice). ${ }^{* *} \mathrm{P}<0.01 \mathrm{I} / \mathrm{R}+\mathrm{NS}$ versus sham-operated mice, $* \mathrm{P}<0.05 \mathrm{I} / \mathrm{R}+\mathrm{QL}$ versus $\mathrm{I} / \mathrm{R}+\mathrm{NS}$.

\section{Results}

QL Treatment Reduces Mortality and Preserves Cardiac Function in Mice after $I / R$ in vivo

The Kaplan-Meier survival curves demonstrated lower mortality in QL treated mice compared with control untreated mice at 7 days after I/R. Indeed, the survival rate of QL treated animals was $80 \%$ and only $52 \%$ mice survived in the control group (log-rank test $P=0.0329$; Fig. 1). Compared with untreated animals, echocardiography showed increased LVEF and LVFS at 1 and 7 days after I/R (Fig. 2A-C). One week after $\mathrm{I} / \mathrm{R}$, the heart weight/body weight (HW/BW) ratios were significantly lower in QL treated mice than in untreated animals (Fig. 2D).

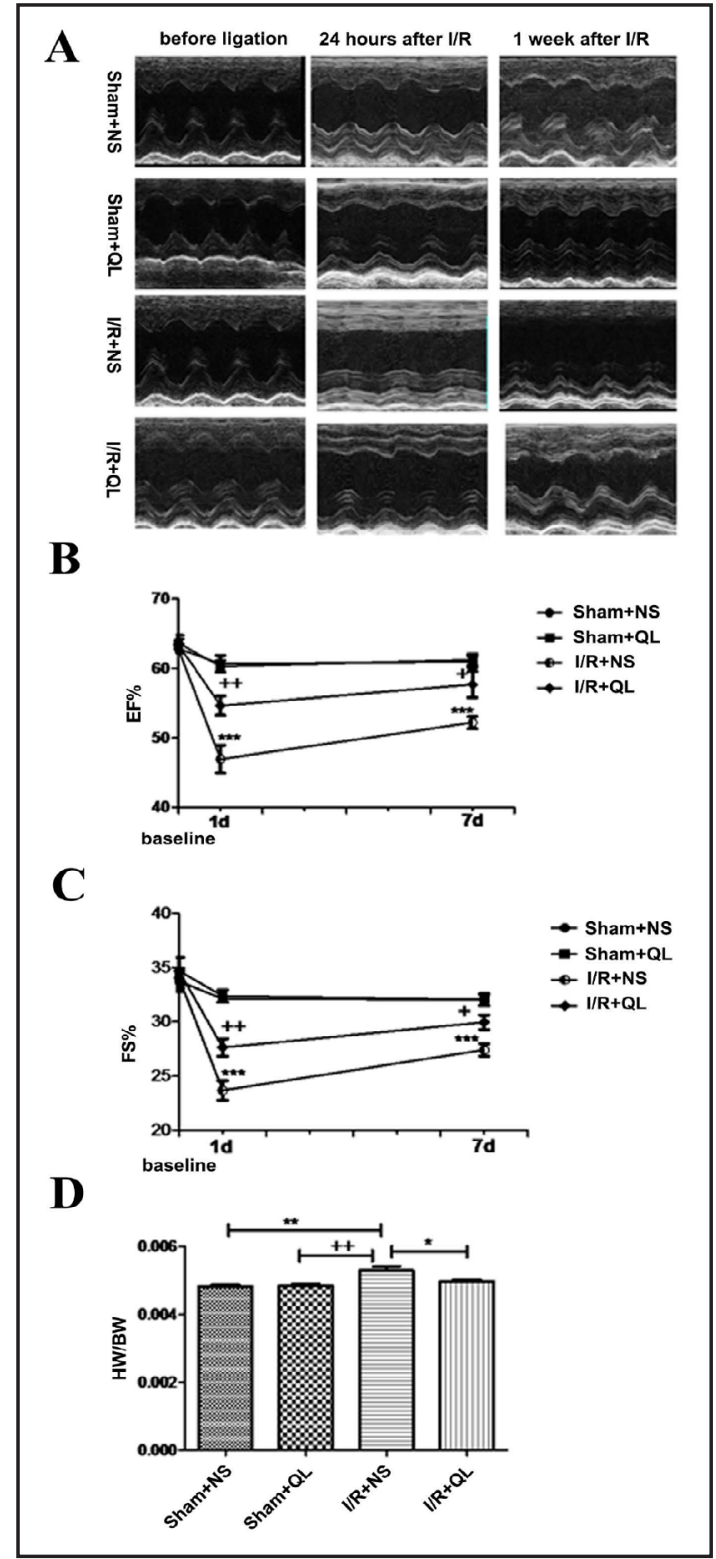

\section{QL Treatment Reduces Infarct Size}

Evans blue and TTC staining methods were used to detect the size of infarct- and noninfarct but at risk areas [27]. The risk areas of the total LV were similar between QL and 


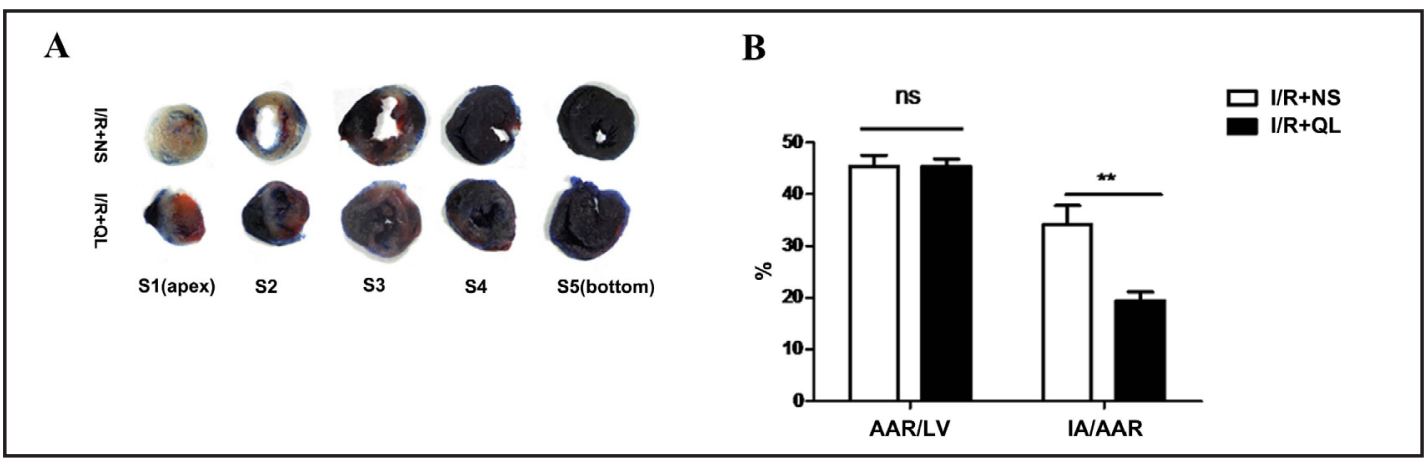

Fig. 3. QL reduces myocardial infarct size after I/R. (A) Hearts were cut transversely into 5-6 slices at 24 hours after I/R. Each slice was stained with Evans blue and TTC. The area at risk (AAR) of myocardium appears red, while the infarct area (IA) is white. (B) Infarct size in QL treated and untreated mice. Infarct size was expressed as the ratio of IA to AAR $(n=10$, QL treated mice; $n=9$, untreated mice) $. * *<0.01$ I/R+QL vs $\mathrm{I} / \mathrm{R}+\mathrm{NS}$.

Fig. 4. QL improves pathological changes in myocardial tissue after I/R. (A) Representative micrographs of $I / R$ mouse hearts after $\mathrm{H} \& \mathrm{E}$ staining. These images demonstrated intense inflammatory infiltration and myocardial cells arranged irregularly after I/R. (B) Representative images of Masson's trichrome-stained hearts from I/R mice. Blue indicates fibrosis. (C) Quantitative analysis of interstitial fibrosis examined by Masson's trichrome staining. Percent fibrosis was determined using Image J software to quantify blue (fibrotic) versus non-blue (non-fibrotic) pixels $(\mathrm{n}=6) .{ }^{* *} \mathrm{P}<0.01 \mathrm{I} /$ $\mathrm{R}+\mathrm{QL}$ vs $\mathrm{I} / \mathrm{R}+\mathrm{NS}$.

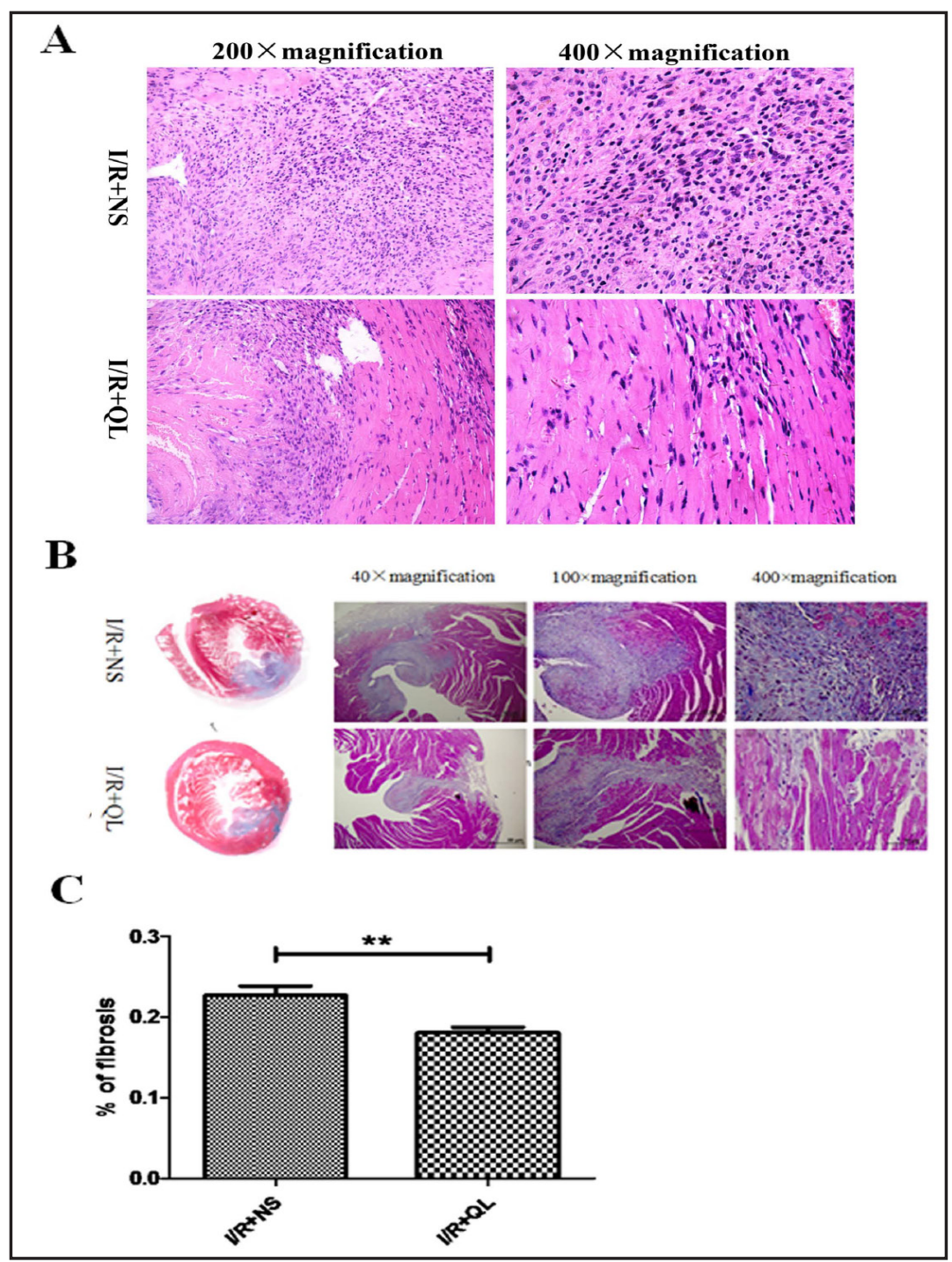

untreated groups. However, treatment with QL resulted in significantly reduced infarct size compared with the untreated group (Fig. 3).

\section{KARGER}


Fig. 5. Effects of QL on mTOR-related signaling molecules in mice after I/R. (A) Representative immunoblots of mTOR signaling molecules in the left ventricular wall. Hearts were harvested at 24 hours after I/R. (B) Densitometric quantification of immunoblots. $\mathrm{n}=6$ mice/group. *P $<0.05, \quad * * \mathrm{P}<0.01$, $* * * \mathrm{P}<0.001$.

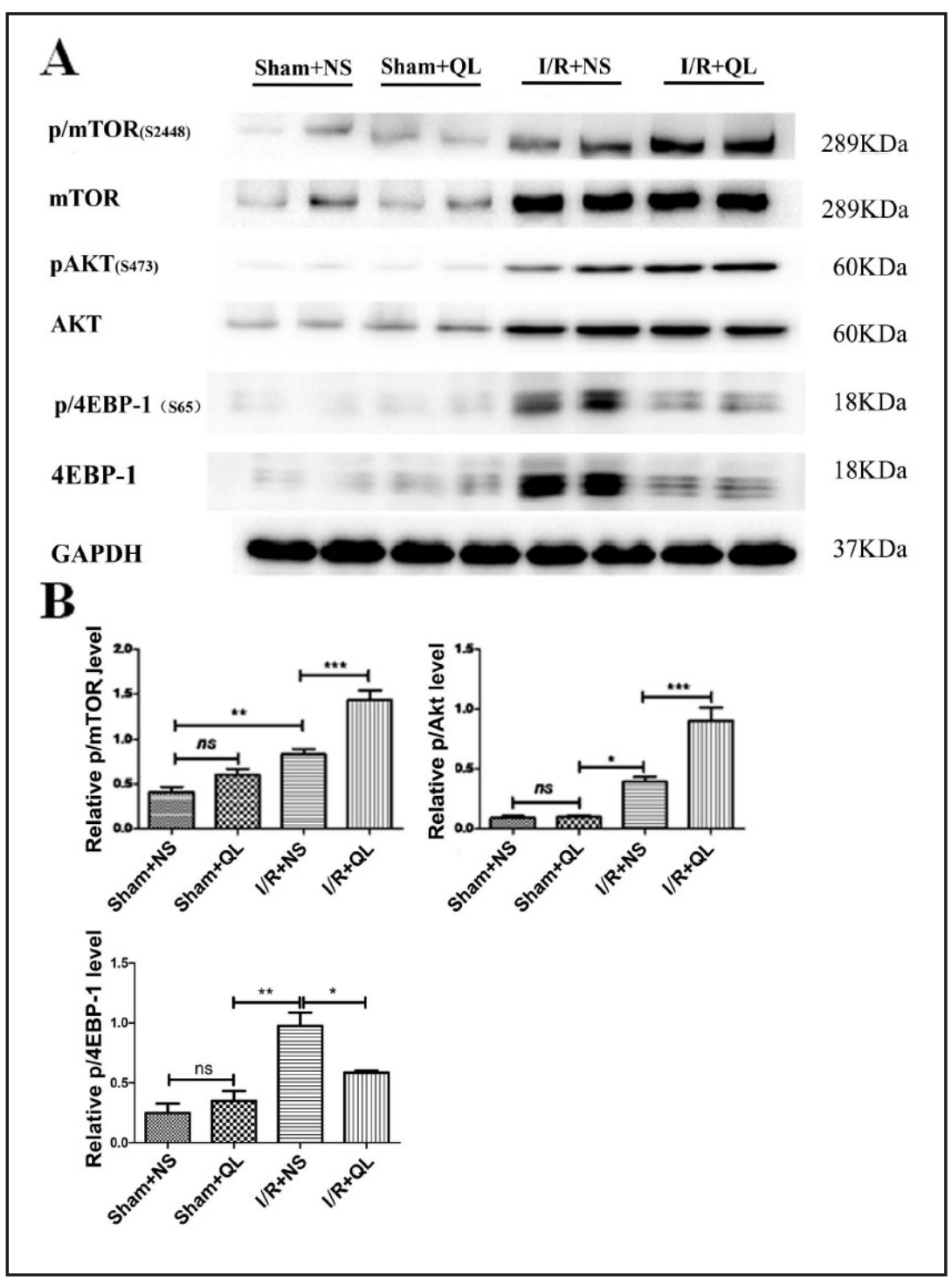

QL Treatment Improves Pathological Changes in Myocardial Tissue

One week after I/R, surviving myocardial cells were found in border zones and arranged irregularly in untreated animals, as shown by H\&E staining. However, most myocardial cells were arranged regularly in the QL treated group (Fig. 4A). In agreement, Masson trichrome staining demonstrated that QL treatment significantly decreased fibrosis and collagen deposition after I/R (Fig. 4B-C).

\section{QL Treatment Activates mTOR Signaling}

We assessed whether QL treatment affected mTOR signaling in the myocardium after I/R. As observed previously $[12,15,16]$, cardiac mTOR signaling can be activated by I/R injury. The expression of phosphorylated mTOR (Ser2448) was significantly higher in the QL treatment group, in comparison with controls (Fig. 5A-B). Similarly, the expression of phosphorylated Akt was significantly higher in QL animals, while phosphorylated 4EBP1 expression was significantly reduced, compared with values obtained for the untreated group (Fig. 5A-B).

Rapamycin Abolishes the Protective Effect of QL against I/R

To further investigate the role of mTOR activation in the therapeutic effect of QL in I/R, pharmacological intervention via rapamycin, an mTOR inhibitor, was used in QL treated I/R mice. The reduced infarct size and improved LVEF and LVFS in QL treated I/R was significantly 


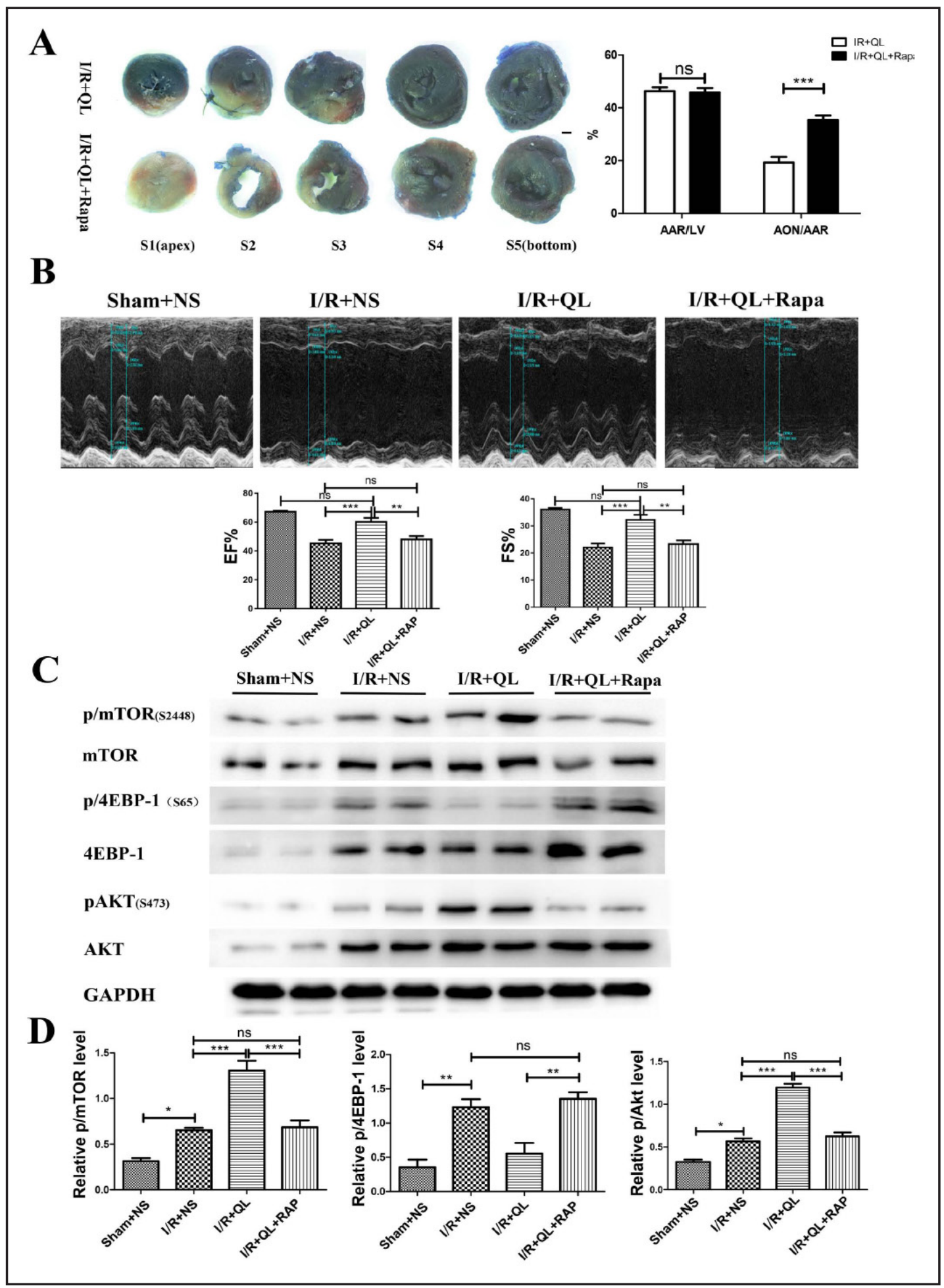

Fig. 6. Rapamycin abolishes the therapeutic effects of QL in mice after I/R. (A) TTC staining and quantitative analysis for infarct size in QL treated I/R mice with and without Rapamycin intervention at 24 hours after I/R. (n=10, I/R+QL mice; n=9, I/R+QL+Rapamycin mice). (B) Echocardiography for LVEF and LVFS at 24 hours after I/R. (n=10, sham+NA mice; $n=10$, I/R+NS mice; n=10,I/R+QL mice; n=9, I/R+QL+Rapamycin mice). (C) Representative immunoblots of mTOR signaling molecules in the left ventricular wall. Hearts were harvested at 24 hours after I/R. D: Densitometric quantification of immunoblots. $\mathrm{n}=6$ mice/group. ${ }^{*} \mathrm{P}<0.05,{ }^{* *} \mathrm{P}<0.01,{ }^{* * *} \mathrm{P}<0.001$.

\section{KARGER}




\section{Cellular Physiology Cell Physiol Biochem 2015;37:454-464 \\ \begin{tabular}{l|l} 
DOI: 10.1159/000430368 & (C) 2015 S. Karger AG, Basel
\end{tabular} \\ and BiOChemistry Published online: August 28, $2015 \quad$ www.karger.com/cpb \\ Zhou et al.: Qiliqiangxin Protects Cardiac Ischemia-Reperfusion Injury}

reversed by Rapamycin (Fig. 6A-B). Meanwhile, the enhanced phosphorylation of mTOR, together with subsequent increased phosphorylated Akt and decreased phosphorylated 4EBP-1 levels in QL treated I/R, were also reversed by Rapamycin (Fig. 6C-D).

\section{Discussion}

Herein, we demonstrated that QL treatment after I/R in mice increased survival rate, decreased infarct size during the acute phase of $\mathrm{I} / \mathrm{R}$ and prevented adverse $\mathrm{LV}$ remodeling in the chronic phase, resulting in the preservation of cardiac function. Moreover, mTOR activation is required for the protective effects of QL in preventing myocardial I/R injury.

A multicenter, randomized, double-blind, parallel-group, placebo-controlled study from our group have shown that in combination with standard treatment, QL further reduced NT-proBNP levels in patients with chronic HF [17]. In addition, QL efficacy against cardiac hypertrophy and remodeling has been demonstrated in several studies [24, 25, 29-36]. Herein, we demonstrated that QL treatment of mice after I/R increased the survival rate, decreased infarct size and decreased fibrosis after I/R, suggesting that QL may also confer cardiac protection against I/R injury.

Previously, it has been reported that mTOR inhibition by rapamycin increases infarct size at $24 \mathrm{~h}$ post-I/R in vivo [37]. However, previous reports have demonstrated that $\mathrm{mTOR}$ inhibition protected the heart in an in vivo MI model using an LAD permanent ligation without reperfusion [38, 39]. A recent report demonstrated that cardiac mTOR overexpression is sufficient to confer substantial cardio-protection against I/R injury and suppress the inflammatory response [16]. The two types of cardiac injury, MI (without reperfusion) and I/R injury, induce cell death with different pathophysiological features; thus, activation of mTOR signaling may have different cardio-protective effects on these two injury types [15]. In line with these studies, our data showed that administration of QL activated the mTOR pathway and reduced mortality as well as infarct size in mice after I/R. Indeed, QL treatment significantly increased p-mTOR levels, induced the expression of phosphorylated Akt and down-regulated phosphorylated 4EBP-1 in mice after I/R, as shown in this study. Moreover, Rapamycin, an mTOR inhibitor, abolished the therapeutic effects of QL in I/R injury. These data indicate that mTOR activation is essential to mediate the protective effect of QL against cardiac I/R injury.

To the best of our knowledge, this is the first study reporting that QL treatment can reduce mouse mortality and infarct size after I/R. Moreover, QL therapy may contribute to improvement of LV function after I/R by inhibiting myocardial fibrosis and enhancing cardiac repair. Indeed we simultaneously observed mTOR activation in QL treated animals, which may be involved in the prevention of myocardial I/R injury. However, several limitations should be highlighted in our study. First, whether a combination of QL with other medications could achieve better therapeutic effects needs further clarification. In addition, a quantitative analysis of leukocyte infiltration and myocardial cells arrangment after QL treatment in I/R is needed in the future.

In conclusion, we have shown that $\mathrm{QL}$ administration has notable benefits on cardiac function and attenuates the progression of heart remodeling after I/R, which is mediated by activation of the mTOR pathway. This study suggests a new application for QL in preventing I/R injury.

\section{Acknowledgments}

This work was supported by the grants from National Natural Science Foundation of China (81370332 and 81170201 to XL Li, 81200169 to JJ Xiao), the Priority Academic Program Development of Jiangsu Higher Education Institutions (PAPD20102013 to XL Li), the National Basic Research Program of China (973 Program, Grant no.2012CB518606), 


\section{Cellular Physiology Cell Physiol Biochem 2015;37:454-464 \begin{tabular}{l|l|l} 
DOI: 10.1159/000430368 & (C) 2015 S. Karger AG, Basel
\end{tabular} and Biochemistry Published online: August 28, $2015 \quad$ www.karger.com/cpb

Innovation Program of Shanghai Municipal Education Commission (13YZ014 to JJ Xiao), Foundation for University Young Teachers by Shanghai Municipal Education Commission (year 2012, to JJ Xiao), Innovation fund from Shanghai University (sdcx2012038 to JJ Xiao), and Program for the integration of production, teaching and research for University Teachers supported by Shanghai Municipal Education Commission (year 2014, to JJ Xiao). Dr XL Li is an Associate Fellow at the Collaborative Innovation Center For Cardiovascular Disease Translational Medicine.

\section{Disclosure Statement}

Dr. Xinli Li received research grants from Shijiazhuang Yiling Pharmaceutical Co. Ltd. All other authors have reported that they have no relationships to disclose.

\section{References}

1 Nallamothu BK, Bradley EH, Krumholz HM: Time to treatment in primary percutaneous coronary intervention. N Engl J Med 2007:357:1631-1638.

2 Prasad A, Stone GW, Aymong E, Zimetbaum PJ, McLaughlin M, Mehran R, Garcia E, Tcheng JE, Cox DA, Grines CL, Gersh BJ: Impact of ST-segment resolution after primary angioplasty on outcomes after myocardial infarction in elderly patients: an analysis from the CADILLAC trial. Am Heart J 2004:147:669-675.

3 Yellon DM, Hausenloy DJ: Myocardial reperfusion injury. N Engl J Med 2007:357:1121-1135.

4 Prasad A, Stone GW, Holmes DR, Gersh B: Reperfusion injury, microvascular dysfunction, and cardioprotection the "Dark Side" of reperfusion. Circulation 2009:120:2105-2112.

5 Pfeffer MA, Braunwald E: Ventricular remodeling after myocardial infarction. Experimental observations and clinical implications. Circulation 1990:81:1161-1172.

6 Vilahur G, Juan-Babot O, Pena E, Onate B, Casani L, Badimon L: Molecular and cellular mechanisms involved in cardiac remodeling after acute myocardial infarction. J Mol Cell Cardiol 2011:50:522-533.

7 Chong ZZ, Shang YC, Maiese K: Cardiovascular disease and mTOR signaling. Trends Cardiovasc Med 2011:21:151-155.

8 Zoncu R, Efeyan A, Sabatini DM: mTOR: from growth signal integration to cancer, diabetes and ageing. Nat Rev Mol Cell Biol 2011:12:21-35.

9 Hara K, Maruki Y, Long X, Yoshino K, Oshiro N, Hidayat S, Tokunaga C, Avruch J, Yonezawa K: Raptor, a binding partner of target of rapamycin (TOR), mediates TOR action. Cell 2002:110:177-189.

10 Jacinto E, Facchinetti V, Liu D, Soto N, Wei S, Jung SY, Huang Q Qin J, Su B: SIN1/MIP1 maintains rictormTOR complex integrity and regulates Akt phosphorylation and substrate specificity. Cell 2006:127:125137.

11 Moschella PC, McKillop J, Pleasant DL, Harston RK, Balasubramanian S, Kuppuswamy D: mTOR complex 2 mediates Akt phosphorylation that requires PKCepsilon in adult cardiac muscle cells. Cell Signal 2013:25:1904-1912.

12 Jonassen AK, Sack MN, Mjos OD, Yellon DM: Myocardial protection by insulin at reperfusion requires early administration and is mediated via Akt and p70s6 kinase cell-survival signaling. Cir Res 2001: 89:11911198.

13 Kis A, Yellon DM, Baxter GF:Second window of protection following myocardial preconditioning: an essential role for PI3 kinase and p70S6 kinase. J Mol Cell Cardiol 2003:35:1063-1071.

14 Vigneron F, Dos Santos P, Lemoine S, Bonnet M, Tariosse L, Couffinhal T, Duplaà C, Jaspard-Vinassa B: GSK3beta at the crossroads in the signalling of heart preconditioning: implication of mTOR and Wnt pathways. Cardiovasc Res 2011:90:49-56.

15 Zhai P, Sciarretta S, Galeotti J, Volpe M, Sadoshima J: Differential roles of GSK-3beta during myocardial ischemia and ischemia/reperfusion. Circ Res 2011:109:502-511.

16 Aoyagi T, Kusakari Y, Xiao CY, Inouye BT, Takahashi M, Scherrer-Crosbie M, Rosenzweig A, Hara K, Matsui T: Cardiac mTOR protects the heart against ischemia-reperfusion injury. Am J Physiol Heart Circ Physiol 2012:303:H75-85. 


\section{Cellular Physiology Cell Physiol Biochem 2015;37:454-464 \begin{tabular}{l|l|l}
\hline DOI: 10.1159/000430368 & (C)15 S. Karger AG, Basel
\end{tabular} and Biochemistry Published online: August 28, 2015

17 Li X, Zhang J, Huang J, Ma A, Yang J, Li W, Wu Z, Yao C, Zhang Y, Yao W, Zhang B, Gao R: A multicenter, randomized, double-blind, parallel-group, placebo-controlled study of the effects of qili qiangxin capsules in patients with chronic heart failure. J Am Coll Cardiol 2013:62:1065-1072.

18 Kang LP, Zhao Y, Yu HS, Liu YX, Xiong CQ, Tan DW, Jia JM, Wang HT, Tian SY, Ma BP: Identification of chemical constituents in qiliqiangxin capsule by UPLC-Q-TOF/MS(E). Yao Xue Xue Bao 2011:46:1231-1236.

19 Wu X, Cao Y, Nie J, Liu H, Lu S, Hu X, Zhu J, Zhao X, Chen J, Chen X, Yang Z, Li X: Genetic and pharmacological inhibition of Rheb1-mTORC1 signaling exerts cardioprotection against adverse cardiac remodeling in mice. Am J Pathol 2013:182:2005-2014.

20 Littlejohns B, Lin H, Angelini GD, Halestrap AP, Suleiman MS: Switching back to normal diet following highfat diet feeding reduces cardiac vulnerability to ischaemia and reperfusion injury. Cell Physiol Biochem 2014:34:1090-1100.

21 Zhang X, Liang X, Lin X, Zhang S, Huang Z, Chen C, Guo Y, Xuan F, Xu X, Huang R: Mechanism of the protective effect of Yulangsan flavonoid on myocardial ischemia/reperfusion injury in rats. Cell Physiol Biochem 2014:34:1050-1062.

22 Yang B, Yang J, Bai J, Pu P, Liu J, Wang F, Ruan B: Suv39h1 protects from myocardial ischemia-reperfusion injury in diabetic rats. Cell Physiol Biochem 2014:33:1176-1185.

23 Qu Z, Xu H, Tian Y, Jiang X: Atorvastatin improves microenvironment to enhance the beneficial effects of BMSCs therapy in a rabbit model of acute myocardial infarction. Cell Physiol Biochem 2013:32:380-389.

24 Wei Y, Liu X, Hou L, Che W, The E, Jhummon MV: Qiliqiangxin affects L type ca(2+) current in the normal and hypertrophied rat heart. Evid Based Complement Alternat Med 2012:2012:131830.

25 Wei Y, Liu X, Wei H, Hou L, Che W, The E, Li G, Jhummon MV, Wei W: The electrophysiological effects of qiliqiangxin on cardiac ventricular myocytes of rats. Evid Based Complement Alternat Med 2013:2013:213976.

26 Bohl S, Medway DJ, Schulz-Menger J, Schneider JE, Neubauer S, Neubauer S, Lygate CA: Refined approach for quantification of in vivo ischemia-reperfusion injury in the mouse heart. Am J Physiol Heart Circ Physiol 2009:297:H2054-2058.

27 Chen P, Pang S, Yang N, Meng H, Liu J, Zhou N, Zhang M, Xu Z, Gao W, Chen B, Tao Z, Wang L, Yang Z: Beneficial effects of schisandrin $B$ on the cardiac function in mice model of myocardial infarction. PLoS One 2013:8:e79418.

28 DeGeorge BR, Jr., Gao E, Boucher M, Vinge LE, Martini JS, Raake PW, Chuprun JK, Harris DM, Kim GW, Soltys S, Eckhart AD, Koch WJ: Targeted inhibition of cardiomyocyte Gi signaling enhances susceptibility to apoptotic cell death in response to ischemic stress. Circulation 2008:117:1378-1387.

29 Kong L, Andrassy M, Chang JS, Huang C, Asai T, Szabolcs MJ, Homma S, Liu R, Zou YS, Leitges M, Yan SD, Ramasamy R, Schmidt AM, Yan SF: PKCbeta modulates ischemia-reperfusion injury in the heart. Am J Physiol Heart Circ Physiol 2008:294:H1862-1870.

30 Tao L, Shen S, Fu S, Fang H, Wang X, Das S, Sluijter JP, Rosenzweig A, Zhou Y, Kong X, Xiao J, Li X: Traditional Chinese Medication Qiliqiangxin attenuates cardiac remodeling after acute myocardial infarction in mice. Sci Rep 2015:5:8374.

31 Ye Y, Gong H, Wang X, Wu J, Wang S, Yuan J, Yin P, Jiang G, Li Y, Ding Z, Zhang W, Zhou J, Ge J, Zou Y: Combination treatment with antihypertensive agents enhances the effect of Qiliqiangxin on chronic pressure overload-induced cardiac hypertrophy and remodeling in male mice. J Cardiovasc Pharmacol 2015:65:628-639.

32 Zhou J, Jiang K, Ding X, Fu M, Wang S, Zhu L, He T, Wang J, Sun A, Hu K, Chen L, Zou Y, Ge J: Qiliqiangxin inhibits angiotensin II-induced transdifferentiation of rat cardiac fibroblasts through suppressing interleukin-6. J Cell Mol Med 2015:19:1114-1121.

33 Xiao H, Song Y, Li Y, Liao YH, Chen J: Qiliqiangxin regulates the balance between tumor necrosis factoralpha and interleukin-10 and improves cardiac function in rats with myocardial infarction. Cell Immunol 2009: 260:51-55.

34 Liu W, Chen JJ, Xu TF, Tian WD, Li Y, Zhang Z, Li W: Qiliqiangxin improves cardiac function in spontaneously hypertensive rats through the inhibition of cardiac chymase. Am J Hypertens 2012:25:250-260.

35 Zou Y, Lin L, Ye Y, Wei J, Zhou N, Liang Y, Gong H, Li L, Wu J, Li Y, Jia Z, Wu Y, Zhou J, Ge J: Qiliqiangxin inhibits the development of cardiac hypertrophy, remodeling, and dysfunction during 4 weeks of pressure overload in mice. J Cardiovasc Pharmacol 2012:59:268-280. 


\section{Cellular Physiology Cell Physiol Biochem 2015;37:454-464}

\begin{tabular}{|l|l|} 
aOI: 10.1159/000430368 & O 2015 S. Karger AG, Basel
\end{tabular}

Zhou et al.: Qiliqiangxin Protects Cardiac Ischemia-Reperfusion Injury

36 Zhang J, Wei C, Wang H, Tang S, Jia Z, Wang L, Xu D, Wu Y: Protective effect of qiliqiangxin capsule on energy metabolism and myocardial mitochondria in pressure overload heart failure rats. Evid Based Complement Alternat Med 2013:2013:378298.

37 Hernandez G, Lal H, Fidalgo M, Guerrero A, Zalvide J, Force T, Pombo CM: A novel cardioprotective p38MAPK/mTOR pathway. Exp Cell Res 2011:317:2938-2949.

38 Buss SJ, Muenz S, Riffel JH, Malekar P, Hagenmueller M, Weiss CS, Bea F, Bekeredjian R, Schinke-Braun M, Izumo S, Katus HA, Hardt SE: Beneficial effects of Mammalian target of rapamycin inhibition on left ventricular remodeling after myocardial infarction. J Am Coll Cardiol 2009:54:2435-2446.

39 Sciarretta S, Zhai P, Shao D, Maejima Y, Robbins J, Volpe M, Condorelli G, Sadoshima J: Rheb is a critical regulator of autophagy during myocardial ischemia pathophysiological implications in obesity and metabolic syndrome. Circulation 2012:125:1134-1146. 